\title{
Impact of Digital Photography and Conventional Method on the Accuracy of Shade Matching in the Esthetic Zone (In Vivo Study)
}

\section{Rawan Ferroukhi ${ }^{1 *}$, Omaima S El Dein El-Mahallawi ${ }^{2}$ and Ahmed Naguib M Hussien ${ }^{3}$}

${ }^{1}$ MSc Student of Fixed Prosthodontics, Division of Fixed Prosthodontics, Faculty of Dentistry, Cairo University, Cairo, Egypt

${ }^{2}$ Professor and Head of Fixed Prosthodontics Department, Faculty of Dentistry, Cairo

University, Cairo, Egypt

${ }^{3}$ Professor of Fixed Prosthodontics Department, Faculty of Dentistry, Cairo University

and Future University, Cairo, Egypt

*Corresponding Author: Rawan Ferroukhi, MSc Student of Fixed

Prosthodontics, Division of Fixed prosthodontics, Faculty of Dentistry, Cairo

University, Cairo, Egypt.
Received: September 02, 2020

Published: September 26, 2020

(C) All rights are reserved by Rawan

Ferroukhi., et al.

\section{Abstract}

Statement of the Problem: Accuracy of shade matching using conventional shade guides is a concern because color perception is highly subjective. Also, entirely relying on the digital method for shade selection can result in a deviation of color interpretation which may have an impact on the device's repeatability and accuracy.

Aim: To evaluate digital photography's accuracy in improving shade matching compared to conventional methods (visual shade guide and spectrophotometer).

Methods: 27 e. max CAD restorations were fabricated for 9 patients. Each received 3 restorations representing three groups for shade selection methods (visual, spectrophotometer and digital photography), to match with the shade of the contra-lateral/adjacent tooth. The patient satisfaction and shade matching were assessed by VAS and modified USPHS criteria respectively. The data obtained was statistically analyzed by Friedman's test performed in categorical data.

Results: In shade matching, there was a statistically significant difference between the three restorations. Restorations in digital photography group showed the highest prevalence of Alpha score followed by Visual 3D Master group then Vita Easyshade group. Group Vita Easyshade was the only restorations with Charlie score. No Delta scores were found. Regarding patient's satisfaction, there was a statistically significant difference between the groups with least satisfaction in the Vita Easyshade group.

Conclusion: Digital photography's impact in shade matching showed validity and reliability over the conventional methods and proved accuracy in terms of shade selection and surface characterization over the visual method. The spectrophotometer showed inconsistency in shade matching.

Keywords: Shade Selection; Shade Matching, Digital Photography, Spectrophotometer, Shade Guide

\section{Introduction}

Shade selection is a crucial part of esthetic dentistry and remains one of the main reasons for esthetic failure. Dentists should effectively deliver this information to the laboratory to create highly aesthetic restorations that seem indistinguishable from the surrounding natural teeth [1]. 
Several methods are available for shade selection. Visual shade matching is the conventional method, in which a commercially available dental shade guide is matched up with the desired tooth. Nevertheless, many factors contribute to perception errors such as different lighting variables and differences in color perception. These factors may alter the ability to achieve the desired results $[1,2]$. Therefore, to eliminate uncontrolled variables during color matching, instrumental tools have been developed. Spectrophotometric methods implement computer calculations based on color science, to allow quantitative evaluation. This method is considered objective and more accurate. Nevertheless, it is restricted to recording color, one point at a time, with absence of standardization of each reading. Moreover, its high price and relatively low performance concerning computer-aided devices are known drawbacks $[2,3]$.

One of the most substantial tools in communicating with patients and dental ceramists is digital photography. Using a digital camera, for color interpretation, is now widely implemented. Photographic images permit the evaluation of multiple points. Also, they help describe surface texture and luster as heavy, moderate, and light to give various combinations for surface characterization $[3,4]$. Therefore, this study was designed to compare available systems to determine whether the digital camera would be an accurate tool for shade matching. The null hypothesis of this study was there would be no significant differences regarding patient satisfaction and shade selection between digital photography and conventional methods (visual shade guide and spectrophotometer).

\section{Materials and Methods}

This study was carried out in Fixed Prosthodontics Department clinics of Faculty of Dentistry, Cairo University, Egypt. Nine patients were involved in this study. Each received three restorations making a total of twenty-seven all-ceramic crowns. Only anterior teeth were included which represented a challenge in terms of shade matching and patient satisfaction [5].

\section{Ethical consideration approval}

The Research Ethics Committee of Cairo university approved this study (approval number 17726), an informed consent in the native language of the participants describing the treatment plan, images' publishing, and collection of results.

\section{Registration}

The trial was registered in the ClinicalTrials.gov under the registration number CEBC-CU-2017-07-17 on date July 2017.

\section{Study design}

This study was a triple blinded quasi-experimental research without randomization.

Inclusion criteria: 1) Age range from 20 to 40 years 2) Mild or moderate discoloration and coronal fracture more than half the tooth 3) Esthetics enhancement of previously placed crowns 4) Endodontically treated teeth 5) Presence of opposite occluding teeth [6].

Exclusion criteria: 1) Poor oral hygiene and compliance 2) Severely discolored teeth 3) Smoking habits 4) Excessive consumption of stain inducing drinks 5) Para-functional habits.

\section{Sample size}

A total sample size of twenty-seven crowns (nine patients) was sufficient, with power $80 \%$ and $5 \%$ significance level. The G power program was used for the sample size calculated [7].

\section{Blinding}

Trial participants, evaluators, and statistician were blinded.

All the clinical procedures were completed by one operator (the researcher). One experienced dental technician fabricated the crowns. Diagnosis, examination, as well as diagnostic photographs, were conducted by the investigator $[6,8]$.

Dental prophylaxis was done before shade selection to remove all debris and stains. Also, it is essential to adjust the environment to be more favorable for shade matching [9].

\section{The shade of the tooth was determined by three methods}

First, visually, using the VITA 3D-Master shade guide (V3D) coinciding with the contra-lateral/adjacent (target) tooth under multiple light conditions: natural daylight, incandescent light, and color corrected light to avoid metamerism with the assistance of five expert prosthodontists that took Ishihara's test for color blindness [10]. All evaluators had no signs of color blindness.

The shade selection was carried out when natural daylight is most balanced with a visible light spectrum $[10,11]$. All bright colors were eliminated from the range of vision (lipstick, tinted eyeglasses). Clear glaze* was used on the shade tab and the natural tooth to keep them hydrated [12]. The patients' mouths were 40 $\mathrm{cm}$ away and at the same level from the evaluator's eyes. Shade tab was situated in the same plane as the target tooth where the incisal third of the shade tab was perpendicular to the incisal portion of 
the target tooth. Both tooth and tab had similar neutral gray background $^{* *}$. Figure 1 shade was selected in 3 points (incisal, middle, and cervical) $[10,11]$. There was no time limit for this procedure; however, the evaluators were requested to rest by staring at a neutral grey zone every ten seconds $[10,14]$.

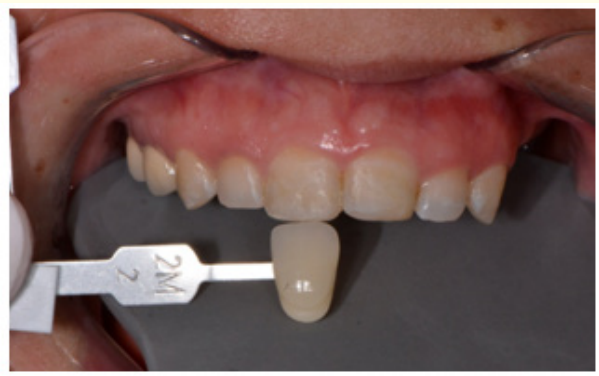

Figure 1: Shade selection under natural day light.

The color corrected light Smile line has a color temperature of $5,500 \mathrm{~K}$, a color rendering index (CRI) of 92 and situated about 10 $\mathrm{cm}$ away from the target tooth [10]. The evaluators were asked to look in the see-thru rectangular window of the color corrected light while standing in front of the target tooth holding the shade tab [15] (Figure 2).

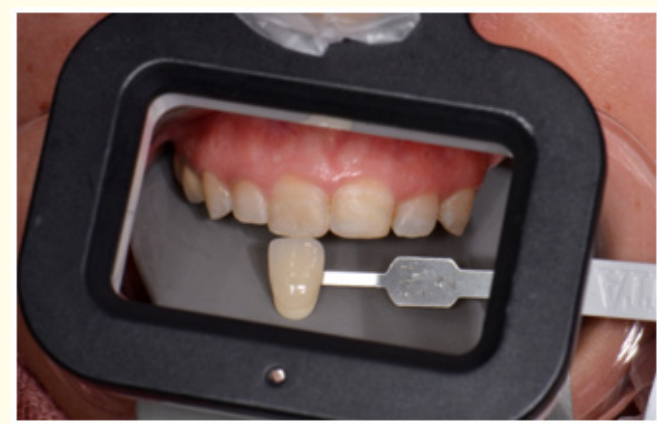

Figure 2: Shade selection using color corrected light.

After the shade selection was achieved, a shade map drawing was sent to the lab [16] (Figure 3).

*IvoclarVivadent Gmbh, Burs, Austria

${ }^{* *}$ Flexipalette Color Match, Smile line, Switzerland.

* VITA, Zahnfabrik, Germany.

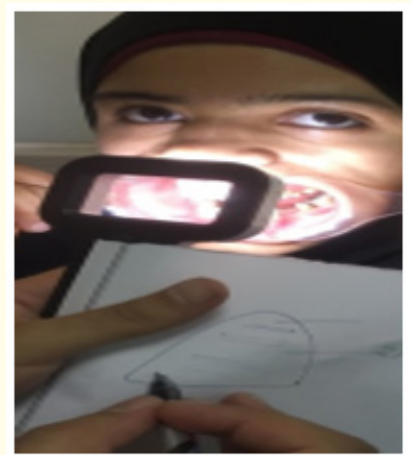

Figure 3: Shade mapping.

Second group, the shade was selected with Vita Easyshade V spectrophotometer [17]* (VE). After placement of the infection control shield on the probing tip, calibration of the device was performed.

Then the mode of shade selection was chosen:

Tooth area measurement (Base shade determination), natural teeth mode. The target tooth was measured by positioning the tip at 90 degrees on the center of the middle 1/3 of labial surface for the exact hue and block shade (Figure 4 and 5).

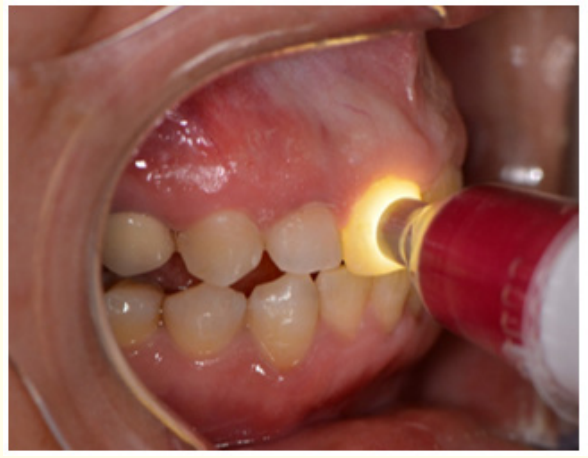

Figure 4: Shade selection. 


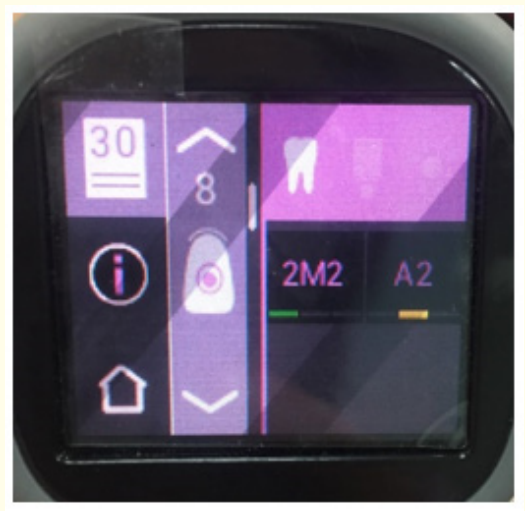

Figure 5: Easyshade V Base shade mode tooth measurement

A 3 spot mode shade mapping was also measured on the (cervical, middle, and incisal 1/3) for overall tooth color (Figure 6). Tooth was illuminated with spectrophotometer light, and the reflected light was analyzed. The display presented the closest shade selected in the classical and 3D Vita shade guide. The measurement was accepted when five successive, similar readings were recorded for each area. Only green (good) matching results were used [18].

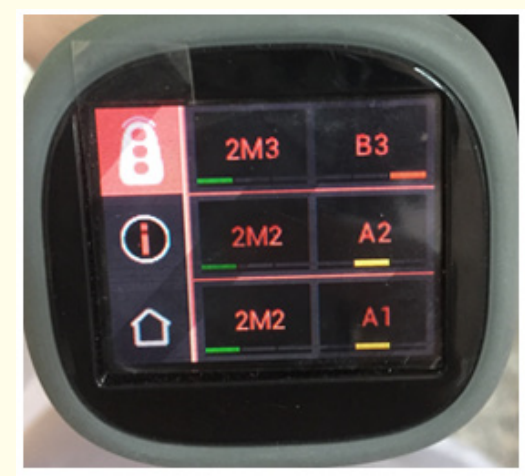

Figure 6: Easyshade V 3 spot shade mapping.

In the third group, the shade was selected with digital photography (D). It was carried out within a standardized environment. A Canon 650D (DSLR) camera was used [6] with a $100 \mathrm{~mm}$ Canon macro lens and lens-mounted Yongnuo macro twin flash. The camera was mounted on a tripod with $70 \mathrm{~cm}$ fixed distance between the lens and the tooth [19]. The patient's head was stabilized with a head stabilizer, and a cheek retractor was placed (Figure 7).

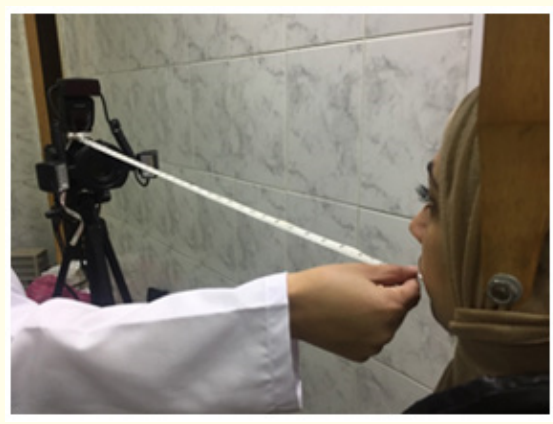

Figure 7: Fixed distance between lens and tooth.

\section{Camera settings}

The camera was set on manual mode with shutter speed $1 / 250$, ISO 200 and custom white balance mode. Aperture for extra-oral photos was set to $\mathrm{f} / 8$, and for intraoral photos $\mathrm{f} / 22$. The lens was set to manual focus. Images captured were saved in RAW file format [12] (Figure 8).

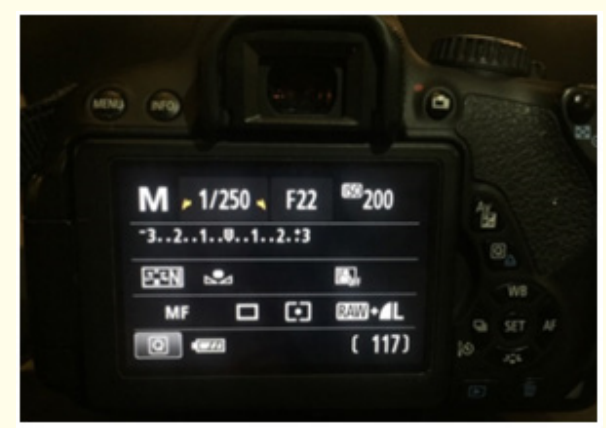

Figure 8: Camera setting.

\section{Flash settings}

Manual mode at 1/4 Flash output for all images except the polarized image.

A polarizer was placed on the twin flash and the lens to reduce the gleam on the teeth by $30 \%$. This enhances visualization and color assessment. Flash settings: was 1:1 and f/32 [20] (Figure 9).

The shade guide and the gray card were present in the image when taking the shade of the tooth. The gray card was utilized for the exposure calibration in Adobe Camera Raw. A WhiBal gray card was used in this study [12,20]. The shade tab and the gray card 
were placed parallel to each other and aligned vertically in the same plane as the natural tooth with the incisal edge resting on the cervical portion of the shade tab [12]. Two or three of the closest shade tabs that were chosen were photographed to be evaluated with Adobe Photoshop.

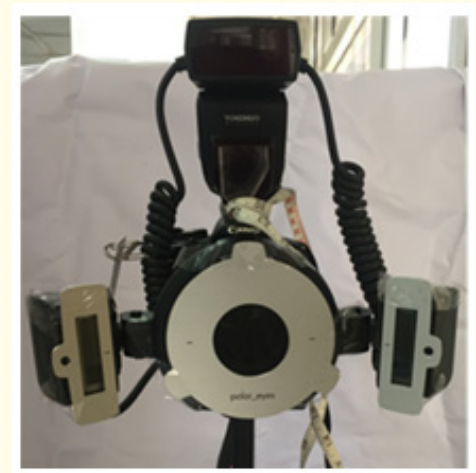

Figure 9: Polarizer placed on the twin flash.

\section{Adobe photoshop steps}

Images were opened into Photoshop (Figure 10). The White balanced tool was selected for the gray card reading of L: 73/74 a: 0 b: 0 . if the gray card didn't read between $73 / 74$ then the exposure was adjusted [12]. The L: reading of the selected shade tab was obtained by placing the white balance tool on the center of middle $1 / 3$ [12]. On the left panel, "INFO" was selected after opening "Window" [12]. Average 5 by 5 image was chosen [12].

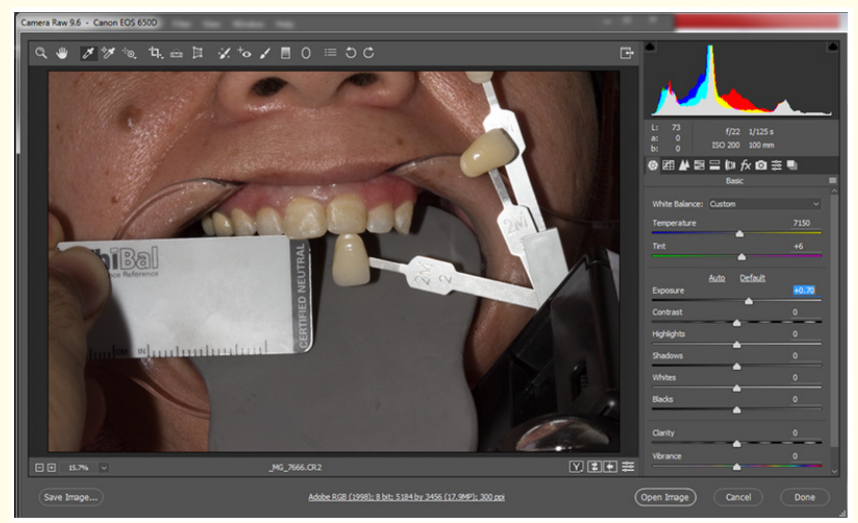

Figure 10: Adobe photoshop steps.

After clicking on that area with the mouse while the shift button remains pressed, the number or reading would appear on the Info panel.

The $\mathrm{L}$ a b color was selected from the Info window by using the small eyedropper tool [11] (Figure 11).

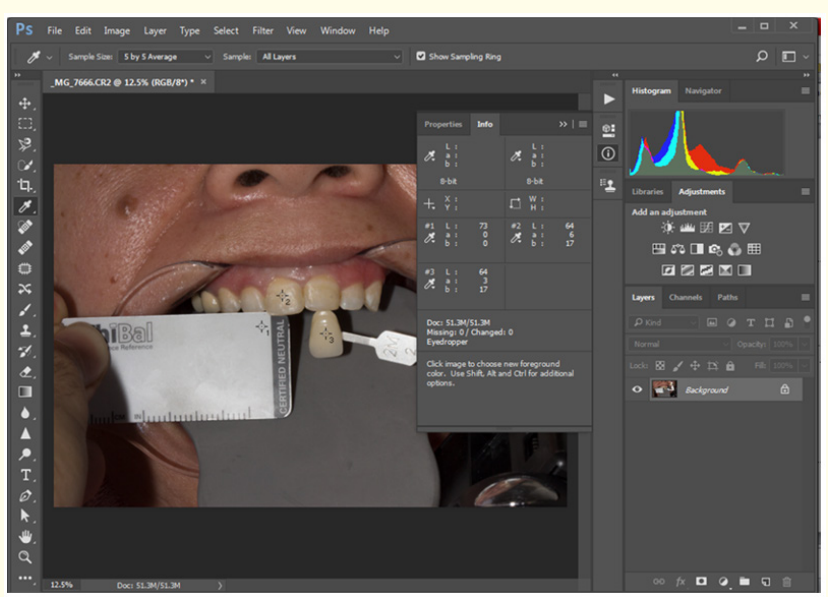

Figure 11: Reading of points in lab.

Click on the "HSB Color" option, which stands for hue, saturation, and brightness [12]. The shade was selected according to the Lab or HSB [12].

Conventional all-ceramic full coverage preparation was performed by preparing the tooth according to manufacturer's guidelines with supra-gingival chamfer finish line of $1 \mathrm{~mm}$ thickness, 2 $\mathrm{mm}$ incisal preparation with the labial surface prepared on two planes which was standardized by the use an index [21,22] (Figure 12 and 13).

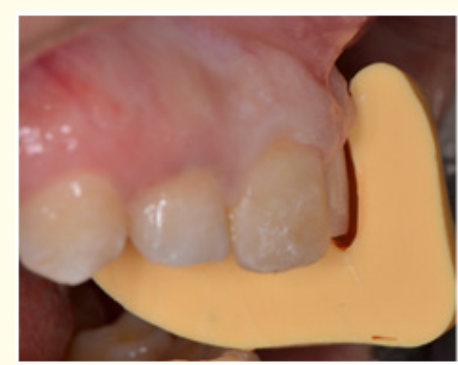

Figure 12: Silicone index showing the amount of reduction labial and incisal.

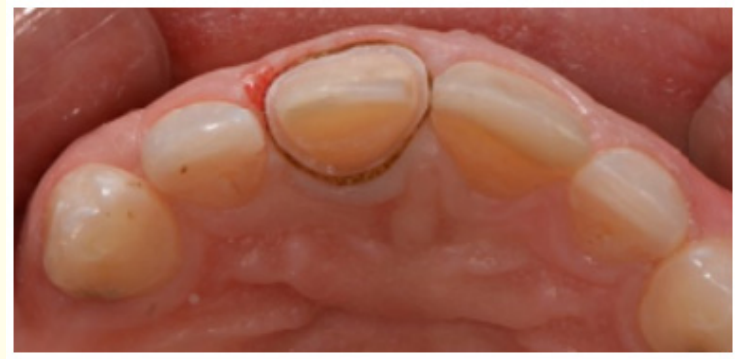

Figure 13: Finishing and rounding of point and line angles of the preparation occlusal view. 
The stump shade of the abutment tooth was selected visually using the Natural Die Material shade guide* under natural daylight and color corrected light [10,23] (Figure 14 and 15).

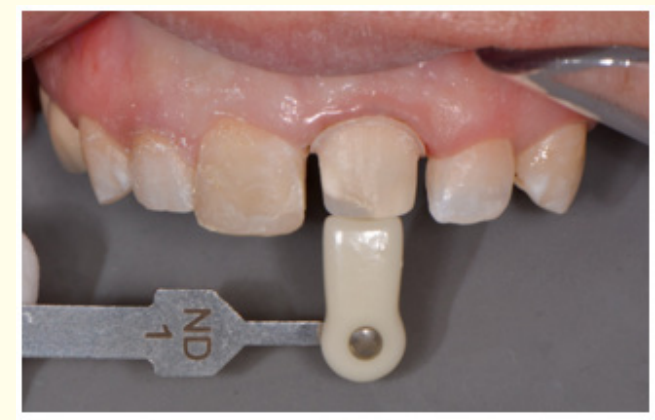

Figure 14: Tooth stump shade selection under natural day light.

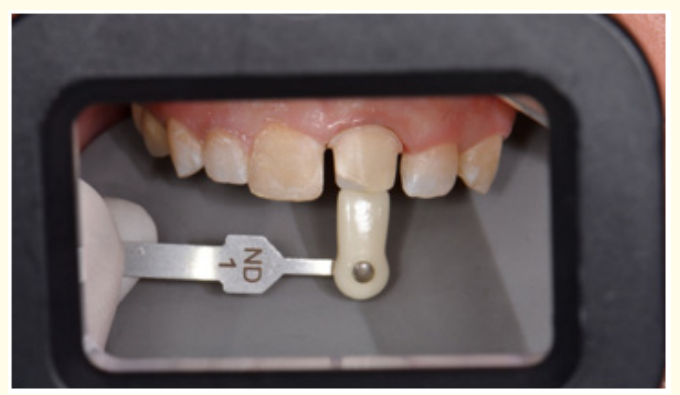

Figure 15: Tooth stump shade selection using color corrected light.

The final impression was taken with addition silicone. Provisional restorations were fabricated and cemented with eugenol free temporary cement. All restorations were fabricated from IPS e.max CAD. After the designing and before the milling, the Ivoclar Vivadent application selected the block shade for the (V3D) group, (D) group while (VE) group was selected using the block feature in one point [24].

The tooth was polished prior to the esthetic try-in which was done under natural daylight and confirmed with color corrected light with the previously selected shade tab in (V3D) group. In (VE) group, the device was used for verification, while in the (D) group; the gray card was used to confirm the shade. After shade confirmation, staining and surface characterization were performed. Dualcured translucent resin cement was used to cement the final restoration after the surface treatment was performed to the tooth and the restoration according to the manufacturer's instruction.

\section{Data collection methods}

\section{Primary outcome}

Patient satisfaction was evaluated using the binary Visual Analogue Scale (VAS) and recorded as satisfactory and unsatisfactory.

Participants were handed a mirror to inspect their teeth and evaluate the three restorations. The final crown was cemented based on the definitive selected restoration by the patient.

\section{Secondary outcome}

Five evaluators assessed in the shade matching of each group using the modified United States public health service (USPHS) criteria:

- Alpha (Excellent): Ideal.

- $\quad$ Bravo (Acceptable): Less than ideal but no modifications required

- $\quad$ Charlie (Acceptable but needs modification): Staining or other shade modifications.

- $\quad$ Delta (Unacceptable): Remake.

Shade matching after staining, glazing, and surface characterization was confirmed with the 3D master Shade tab, Vita Easyshade $\mathrm{V}$ spectrophotometer, and digital camera. Data were recorded, tabulated then statistically analyzed (Figure 16-18).

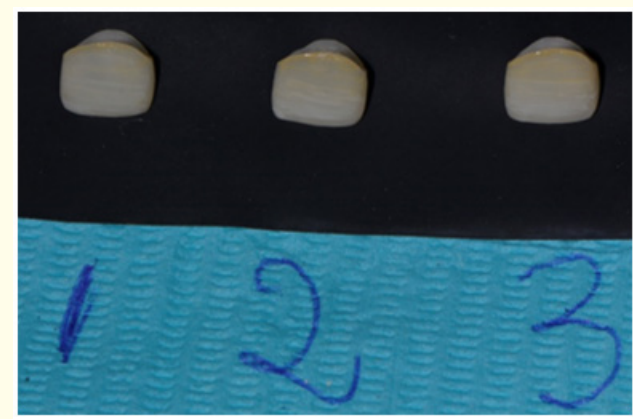

Figure 16: The three crowns placed on the bracket table for assessment.

*IPS Natural Die Material Guide, Ivoclar Vivadent. 


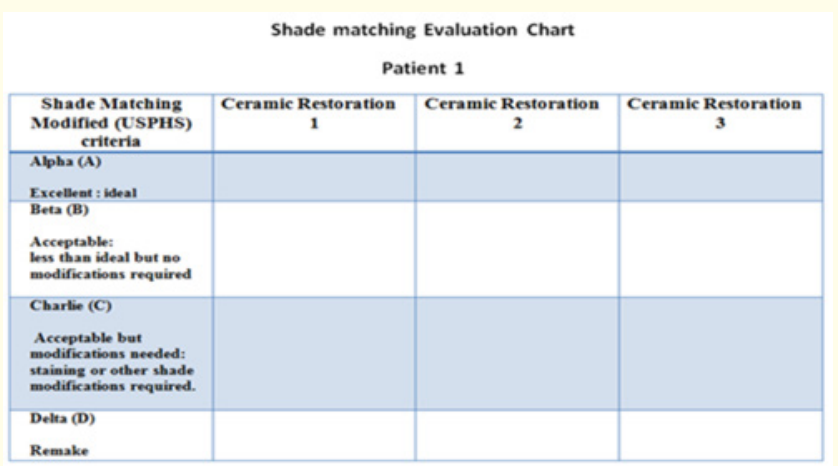

Figure 17: Evaluation chart.
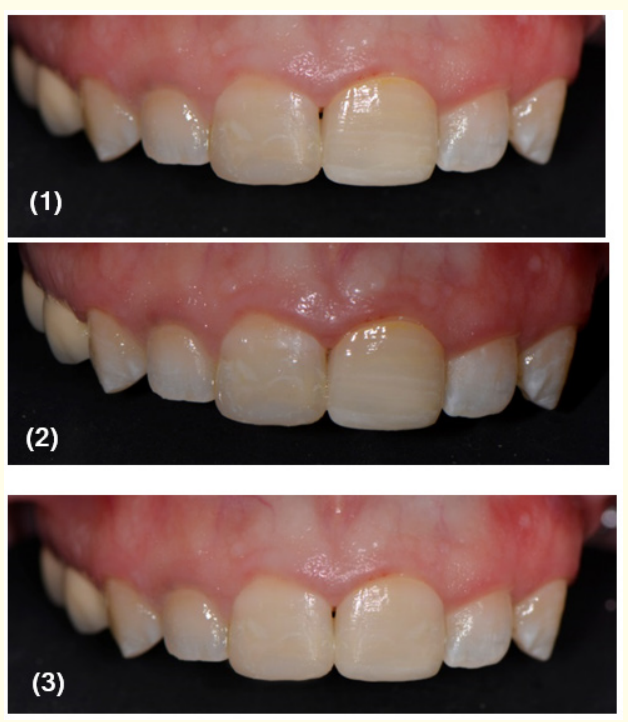

Figure 18: Evaluation of shade matching. (1) Visual shade tab group. (2) Easy shade V group. (3) Digital Camera group.

\section{Results}

\section{Statistical analysis}

Qualitative data were demonstrated as frequencies and percentages. The data obtained for evaluating the outcomes were statisti- cally analyzed using Friedman's test and used to compare between the three groups. The significance level was determined to be at $\mathrm{P}$ $\leq 0.05$. Statistical analysis was performed with IBM SPSS Statistics for Windows, Version 23.0. Armonk, NY: IBM Corp. Sample size ( $\mathrm{n}=$ 9) was enough to detect large effect sizes for main effects and pairwise comparisons, and the satisfactory level of power set at $80 \%$ and a 95\% confidence level [7].

\section{Demographic data}

This study was carried out on nine participants; all of them were females. The mean and standard deviation values for age were 30 years with a minimum of 20 and a maximum of 40 .

\section{Shade matching evaluation}

The results of a comparison between the three groups were presented in table 1 and figure 19. There was a statistically significant difference between the three restoration types $(P$-value $=$ 0.002 , Effect size $=0.722$ ). Six restorations of Group (V3D) showed (66.74\%) Alpha score, while three restorations showed (33.3\%) Bravo score.

All restorations of Group (D) showed a (100\%) Alpha score. While for restorations of Group (VE): two restorations showed (22.2\%) Alpha score, three restorations showed (33.3\%) Bravo score, and four restorations showed (44.5\%) Charlie score. All groups showed (0\%) Delta score.

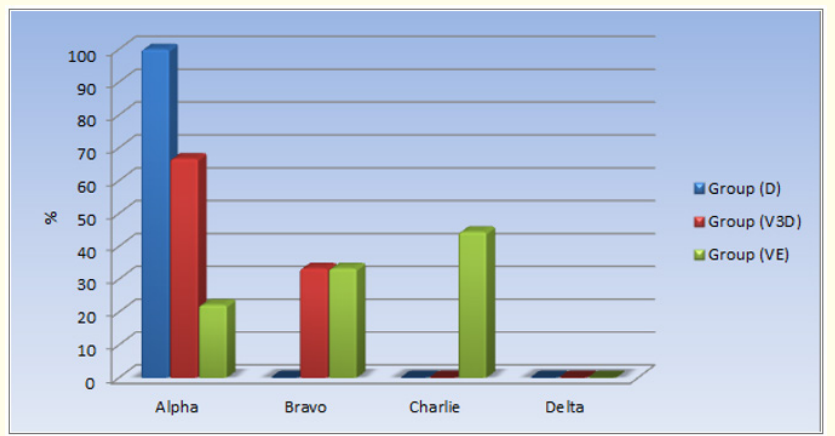

Figure 19: Bar chart representing shade matching scores.

\begin{tabular}{|l|c|c|c|c|c|c|c|c|}
\hline \multirow{2}{*}{ Scores } & \multicolumn{2}{|c|}{ Group (D) (n = 9) } & \multicolumn{2}{|c|}{ Group (V3D) (n = 9) } & Group (VE) (n = 9) & \multirow{2}{*}{ P-value } & $\begin{array}{c}\text { Effect size } \\
\text { (w) }\end{array}$ \\
\cline { 2 - 7 } & $\mathbf{N}$ & $\mathbf{\%}$ & $\mathbf{N}$ & $\mathbf{\%}$ & $\mathbf{N}$ & $\mathbf{\%}$ & & \multirow{2}{*}{0.722} \\
\hline Alpha & 9 & 100 & 6 & 66.7 & 2 & 22.2 & $0.002 *$ & \\
\hline Bravo & 0 & 0 & 3 & 33.3 & 3 & 33.3 & & \\
\cline { 1 - 7 } Charlie & 0 & 0 & 0 & 0 & 4 & 44.5 & & \\
Delta & 0 & 0 & 0 & 0 & 0 & 0 & & \\
\hline
\end{tabular}

Table 1: Frequencies (n), percentages (\%) and results of Friedman's test for comparison between shade matching evaluation scores of the three groups.

*: Significant at $\mathrm{P} \leq 0.05$. 


\section{Patient satisfaction}

The results of patient's satisfaction associated with restorations is highlighted in table 2 and figure 20.

All restorations of (D) group and (V3D) group restorations were $(100 \%)$ satisfactory for the patients. Regarding the restorations of the group (VE), 55.6\% were satisfactory, and $44.4 \%$ were not satisfactory. There was a statistically significant difference between the three groups $(P$-value $=0.018$, Effect size $=0.444)$.

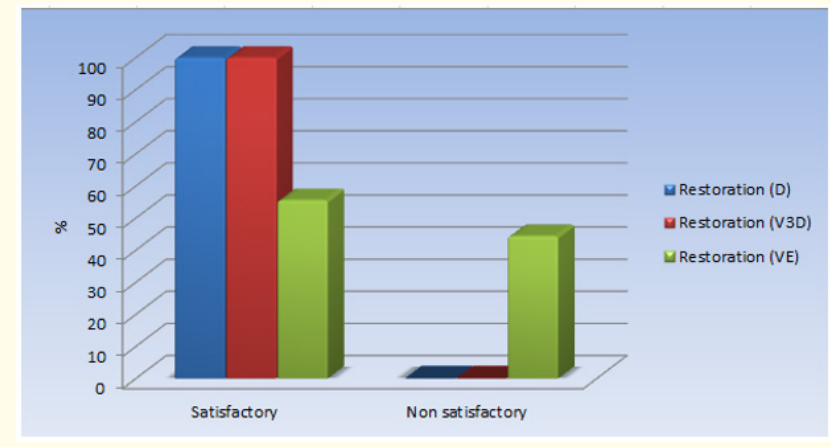

Figure 20: Bar chart representing patient satisfaction.

\begin{tabular}{|c|c|c|c|c|c|c|c|c|}
\hline \multirow[t]{2}{*}{ Satisfaction } & \multicolumn{2}{|c|}{$\begin{array}{l}\text { Restoration (D) } \\
\qquad(n=9)\end{array}$} & \multicolumn{2}{|c|}{$\begin{array}{l}\text { Restoration (V3D) } \\
\qquad(\mathrm{n}=9)\end{array}$} & \multicolumn{2}{|c|}{$\begin{array}{l}\text { Restoration (VE) } \\
\qquad(n=9)\end{array}$} & \multirow[t]{2}{*}{ P-value } & \multirow[t]{2}{*}{$\begin{array}{l}\text { Effect size } \\
\text { (w) }\end{array}$} \\
\hline & $\mathbf{N}$ & $\%$ & $\mathbf{N}$ & $\%$ & $\mathbf{N}$ & $\%$ & & \\
\hline Satisfactory & 9 & 100 & 9 & 100 & 5 & 55.6 & \multirow[t]{2}{*}{$0.018^{*}$} & \multirow[t]{2}{*}{0.444} \\
\hline Unsatisfactory & 0 & 0 & 0 & 0 & 4 & 44.4 & & \\
\hline
\end{tabular}

Table 2: Frequencies (n), percentages (\%) and results of Friedman's test for comparison between satisfactions with the three restoration types.

$*$ : Significant at $\mathrm{P} \leq 0.05$.

\section{Discussion}

In the present study, all teeth included were anterior teeth due to their presence in the esthetic zone as they are very challenging in terms of shade matching and patient satisfaction [5]. Starting by the visual shade matching, Vitapan 3D-Master shade guide was chosen in this study due to its extensive use in dental clinics. Also, the recommendation of several studies about the shade tabs' color being evenly distributed, enables accurate shade matching with the natural teeth. Li., et al. [25] and Zenthöfer., et al. [26], who assessed the commercial shade guides, reported that the Vitapan 3D-Master shade guide was efficient and facilitated the shade selection in the clinic because the shade tabs were more evenly spaced in the color spectrum.

Illumination also plays a significant role in producing an accurate shade selection; therefore, a color corrected light was used with $5500 \mathrm{~K}$ color temperature as an adjunctive tool for optimum shade selection. Illumination in the dental office is a mixture of daylight and light generated by the dental chair lamp. Since these conditions are incredibly variable, light correcting sources have been recommended to be used during shade selection in dentistry
[27]. According to Curd., et al. [28], dental students provided better shade matching outcomes using a color corrected light than natural light. Also, Joiner [29] and Clary., et al. [15] found that shade matching results with handheld lights were superior to those acquired with a viewing booth or natural daylight.

Earlier, Fondriest J [16] stated that most humans have eye dominance, and thus, it was not recommended to place the shade guide on the side of the tooth; otherwise, one eye would preferentially perceive the shade, giving inaccurate evaluation. He also reported that shade tabs should be positioned in the same plane of the tooth, neither in front of it as it would appear lighter, nor behind it as it would appear darker. Pitel ML [30], suggested placement of the shade tab below the tooth, with the incisal edge towards the tooth's incisal edge, which was considered more favorable for reflection of the light perceived by the eye. The best background for hue and chroma selection is gray because neutral gray has no complementary color, making it restful to the retinal cones [16].

Moreover, McLaren., et al. [12] stated that to control the shade selection environment, the teeth needed to stay hydrated, as de- 
hydration causes the teeth to appear brighter. Using cheek retractors causes the saliva to dry quickly; thus, a medium-viscosity clear glaze liquid was used to wet the teeth and shade guide. This procedure was performed in both groups (V3D) and group (D) during shade selection.

In the present study, trained and experienced prosthodontists were chosen for color evaluation. According to Jaju., et al. [31] and Ristic., et al. [32] clinical experience and knowledge of color science could enhance the performance clinically in complex color matching.

3 females and 2 males prosthodontist evaluators were selected in this study, there was no significant gender influence on shade matching ability as Clary., et al. [15] and Joshi and Acharya J [33] found.

Because the consistency and reliability of visual shade matching were questioned, Vita Easyshade V was used in this study, according to Kim-Pusateri., et al [17]. Several brands were tested against many different types of shade guides, in simulated conditions, multiple times. Reliability results were similar; all the tested instruments scored in the $90 \%$ range. Accuracy, however, showed statistically significant differences ranging from 60\% - 90\%. Only the Vita Easy Shade scored within the $90 \%$ range for both accuracy and reliability.

After performing a systematic review of visual and instrumental measurements for tooth shade matching, Chen., et al. [34] found that the instrumental method provided the most precise and accurate shade-matching outcomes. Moreover, to improve the esthetic outcome, Igiel., et al. [35] suggested using color matching instruments as supplementary tools in daily dental practice.

In recent studies, digital photography plays an increasingly important role in everyday dental practice as a useful communication tool between the dentist and the laboratory. Several (DSLR) cameras are available in the market where the most commonly used camera brands in dental photography were Canon at $48 \%$ with a macro lens of a $100 \mathrm{~mm}$ and Nikon at $52 \%$ with a macro lens of $105 \mathrm{~mm}[16]$.

A Canon DSLR camera was used in this study. The settings were in accordance with Culic., et al. [36] that had the images standardized by adjusting the dental camera settings as follows: manual mode 'M' which allowed the manual control of exposure's param- eters, F22 aperture value, and 1/200 Shutter speed, manual focus, maintaining a constant distance between the lens and the object; white balance - flash (color temperature 6500K); ISO 100 and flash mode manual at $1 / 4$ power ratio. In another study, Oh., et al. [37], used the Nikon D70s DSLR camera set on manual mode (M) program, with a $105 \mathrm{~mm}$ macro lens at 1:1 magnification. The shutter speed was set at $1 / 125$ seconds with the aperture of $F / 20$. At an angle of 45 degrees, a bilateral flash was fixed to the front of the camera lens. For standardization purposes, a fixed distance between the teeth and the camera was set before taking an image. Unfixed camera distance was considered as uncontrolled environmental factors. In this experiment, the shooting distance was set to $70 \mathrm{~cm}$ in accordance to Miyajiwala's., et al. study [19].

Earlier in 2009, Ahmed., et al. [38] reported that the light output of electronic flashes was corrected to 'photographic daylight' with a color temperature of 5,500 K. Photographic daylight, instead of natural daylight, is the ideal light for photography, automatically adjusted by the camera unlike natural daylight which may change according to external uncontrolled conditions. Natural daylight produced a continuous, seamless spectrum while electronic flash produces a discontinuous spectrum. Therefore, in this study, the light of the twin flash was considered sufficient to ensure ideal conditions for shade selection and the use of an external color corrected light was not necessary.

Also, McLaren and Chang [39] compared different camera flashes and reported that the ring flash tends to flood the scene with light, giving a flat image. The surface texture and translucency couldn't be captured with this type of flash. A dual-flash can record surface texture detail and contour and is best used for anterior teeth.

McLaren., et al. [12] used a DSLR camera, a macro lens and a twin flash suitable for macro photography tethered into Adobe Photoshop CC using the RAW format.

According to the manufacturer and Liu, 2019, the YN-24EX twin flash was a great alternative to the highly priced Canon MT-24EX macro twin flash [40].

In accordance with Hein and Zangl [20] and McLaren., et al. [12], the use of a standardized gray reference card promised to overcome the limitation of visually perceivable effects in the digital images through a remapping process of the original RAW image to a defined standard. 
They have also found the most accurate card to be the WhiBal ${ }^{\circledR}$ card, which had an L value between 74 and 75 as measured in the CIELAB. Determining the value sometimes could be challenging. A polarized light filter would eliminate the reflected light making it easier to define wither the brightness is caused by low chroma or high surface reflectivity. In other words, the cross-polarized filters eliminate specular reflections that conceals the fine details of the tooth, providing a glare-free image. A polarized image improved the visualization of the dentin base shade and fine enamel features, providing a chromatic map [16].

Hein and Zangl [20] and McLaren., et al. [12] analyzed the shade using a polarized filter sheet for cross-polarization, which revealed intrinsic shade variations of natural teeth.

Shade matching results revealed that the digital camera and visual method outcomes were comparable. The only difference was in the surface characterization as in the (D) group in which sending a photograph to the laboratory made the restoration look identical to the tooth in comparison to shade map drawing in (V3D) group where some details may be missed which had a direct impact on the results.

The Alpha score results obtained might be reasonable since it is the combined reflection of the meticulous shade matching protocol. The performance of (VE) group in cases of monochromatic teeth was accurate and reliable, while in 4 restorations that had surface characterization and surface texture, the final outcome was affected.

In agreement with Anand., et al. [41], this study revealed that digital photography was as accurate as the most commonly used method for shade selection and that a DSLR camera with Adobe Photoshop could be a replacement to a spectrophotometer in acquiring ' $\mathrm{L}$ ' and ' $\mathrm{b}$ ' values precisely.

Schropp [42] reported no statistical differences between visual and digital measurements. In contrast, Jarad., et al. [43] reported that the observers' shade matching performance was significantly higher with the computer method compared to the conventional one, and also stated that digital imaging reveals more information related to the tooth color, shape, and particular characteristics that the technician may use to fabricate an ideal restoration.

McLaren., et al. [12] reported difficulty in describing a complex, multi-layered, multi-textured, 3D color scheme with a 2D shade guide system. A precise clinical photograph could register various details that would be un-noticeable by the eyes.
Hein., et al. [44] stated that the shade matching performance of the observer was remarkably improved with the computer method using the digital camera with a significant variability in the observer's matching capability when selected visually.

Coinciding with the results in this present study, Kim [18] stated that while two dissimilar EasyShade V devices showed high repeatability of CIE $\mathrm{L}^{*}, \mathrm{a}^{*}$ and $\mathrm{b}^{*}$ measurements, they provided inconsistent color values and shade for the individual tooth caused by tooth curvature along with the concise aperture size which leads to edge loss effect. The translucency and surface characteristics could influence the repeatability and accuracy of the EasyShade V. Additionally, it wasn't often convenient to place the aperture on the same area each time.

Contradicting results according to a systematic review, Chen., et al. [34] reported that nine out of seventeen studies showed more precise outcomes using a spectrophotometer. While, three studies showed relatively lower reproducibility, caused by device-dependent biases. Regarding the eight studies inspecting the shade matching accuracy, three studies reported higher accuracy rates. In some studies, the spectrophotometer is considered a gold standard for shade selection.

This was explained by Kim [18], who reported that shade matching accuracy, using spectrophotometer devices, in the in vitro studies were higher than in vivo.

Zeighama., et al. [45] pointed out several factors affecting the final color of the restorations including porcelain layering technique [46], dental ceramic type, primary shade [47], ceramic brand [48], thickness of veneering ceramic [49] and firing cycles [50,51]. Additionally, the cement type, color and thickness also contributed to the final color.

Regarding lithium disilicate CAD crowns, Fasbinder., et al. [52] reported that Alpha scores were recorded even after two years.

Moreover, Rauch., et al. [53] found that the color of all lithium disilicate crowns recorded only Alpha scores after 6 and 10 years.

\section{Patient satisfaction}

The $100 \%$ patient satisfaction of both (D) and (V3D) group could be justified by the sensitivity of dentists towards the identification of the deviations in the shade than laypeople. While in (E) group, only 5 patients were satisfied, and 4 patients were not satisfied because these restorations were, according to the patients, more yellowish. It was coinciding with the results of shade matching recorded previously by the 5 evaluators. 
In 2017, Ballard., et al. [54] added that participants reported high satisfaction which was influenced by the lightness of the crown's shade.

In contradiction to our results, Shah., et al. concluded that the general patient satisfaction was moderate [55]. They clarified that the level of education of the patients had an effect on the results.

Also, Ballard [54] reported that dental school patients were aware that students were performing the restorations. This could slightly lower their expectation for the restorations' shade compared to an analogous private practice patient.

Finally, the hypothesis was rejected as digital photography group was graded Alpha. It was found to be an accurate method that can be used with the visual method or as an alternative for spectrophotometer in shade matching.

The limitations of this study were:

- $\quad$ Further clinical studies on larger sample size with the use of different softwares available in the market are recommended.

- A practitioner, with inadequate knowledge of Adobe Photoshop can encounter difficulties in shade selection.

- $\quad$ Patients with severely discolored teeth and different processing techniques as layering or cut-back may affect the final outcome.

\section{Conclusion}

- Digital photography method proved accuracy in terms of shade selection and surface characterization over the conventional visual method.

- $\quad$ Spectrophotometer, as an objective method, showed inability to capture the various tooth surface characterization and recorded excellent results in case of monochromatic teeth.

\section{Bibliography}

1. John MT., et al. "Oral health-related quality of life in patients treated with fixed, removable, and complete dentures 1 month and 6 to 12 months after treatment". International Journal of Prosthodontics 17 (2004): 503-511.

2. Grossmann AC., et al. "Treatment with double crown-retained removable partial dentures and oral health-related quality of life in middle- and high-aged patients". International Journal of Prosthodontics 20 (2007): 576-278.
3. Baldwin DC. "Appearance and aesthetics in oral health". Community Dentistry and Oral Epidemiology 8 (1980): 244-256.

4. Samorodnitzky-Naveh GR., et al. "Patients' satisfaction with dental esthetics". Journal of the American Dental Association 138 (2007): 805-808.

5. Baharin SA., et al. "Anterior tooth shade selection procedure: influence of light sources and patient's position". Sains Malaysiana 42.1 (2013): 7-11.

6. McLaren EA and Schoenbaum T. "Digital photography enhances diagnostics, communication, and documentation". Compendium of Continuing Education in Dentistry (Jamesburg, NJ: 1995) 32 (2011): 36-38.

7. Jarad FD., et al. "The use of digital imaging for colour matching and communication in restorative dentistry". British Dental Journal 199.1 (2005): 43-49.'

8. Goodlin R. "Photographic-assisted diagnosis and treatment planning". Dental Clinics 55.2 (2011): 211-227.

9. Shammas M and Alla RK. "Color and shade matching in dentistry". Trends in Biomaterials and Artificial Organs 25.4 (2011): 172-175.

10. ABOU-STEIT Sameh., et al. "Evaluation of patient satisfaction and shade matching of Vita Suprinity versus lithium disilicate (E-max) ceramic crowns in the esthetic zone: a randomized controlled clinical trial". F1000Research 8.371 (2019): 371.

11. Fairchild MD and Reniff L. "Time course of chromatic adaptation for color-appearance judgments". Journal of the Optical Society of America A: Optics, Image Science and Vision 12 (1995): 824-833.

12. McLaren EA., et al. "A technique using calibrated photography and Photoshop for accurate shade analysis and communication". Compendium of Continuing Education in Dentistry 38 (2017): 106-113.

13. Özat P B., et al. "Repeatability and reliability of human eye in visual shade selection". Journal of Oral Rehabilitation (2013).

14. Parameswaran V., et al. "Comparison of accuracies of an intraoral spectrophotometer and conventional visual method for shade matching using two shade guide systems". The Journal of the Indian Prosthodontic Society 16 (2016): 352-358.

15. Clary JA., et al. "Influence of light source, polarization, education, and training on shade matching quality". Journal of Prosthetic Dentistry 116 (2016): 91-97. 
16. Fondriest J. "Shade matching in restorative dentistry: the science and strategies". International Journal of Periodontics and Restorative Dentistry 23.5 (2003): 467-480.

17. Kim-Pusateri S., et al. "Reliability and accuracy of four dental shade-matching devices". The Journal of Prosthetic Dentistry 101.3 (2009): 193-199.

18. Kim HK. "Evaluation of the repeatability and matching accuracy between two identical intraoral spectrophotometers: an in vivo and in vitro study". The Journal of Advanced Prosthodontics 10.3 (2018): 252-258.

19. Miyajiwala J S., et al. "Comparison of photographic and conventional methods for tooth shade selection: A clinical evaluation". The Journal of the Indian Prosthodontic Society 17.3 (2017): 273.

20. Hein S and Zangl M. "The use of a standardized gray reference card in dental photography to correct the effects of five commonly used diffusers on the color of 40 extracted human teeth". International Journal of Esthetic Dentistry 11.2 (2016): 246-259.

21. Etman M K and Woolford MJ. "Three-year clinical evaluation of two ceramic crown systems: A preliminary study". Journal of Prosthetic Dentistry 103 (2010): 80-90.

22. Chaiyabutr Y and Kois JC. "Effect of abutment tooth color, cement color, and ceramic thickness on the resulting optical color of a CAD/ CAM glass-ceramic lithium disilicate- reinforced crown". Journal of Prosthetic Dentistry 105 (2011): 83-90.

23. Shillingburg HT., et al. "Fundamentals of fixed prosthodontics". (2012): 149-163.

24. Kalantari MH., et al. "Evaluation of accuracy of shade selection using two spectrophotometer systems: Vita Easyshade and Degudent Shadepilot". European Journal of Dentistry 11 (2007): 196-200.

25. Li Q., et al. "In vivo spectroradiometric evaluation of color matching errors among five shade guides". Journal of Oral Rehabilitation 36 (2009): 65-70.

26. Zenthöfer A., et al. "Selecting VITA Classical Shades with the VITA 3D-Master Shade Guide". International Journal of Prosthodontics 27 (2014): 376-382.

27. Gasparik, C., et al. "Influence of light source and clinical experience on shade matching". Clujul Medical 87.1 (2014): 30.
28. Curd F M., et al. "Comparison of the shade matching ability of dental students using two light sources". The Journal of Prosthetic Dentistry 96.6 (2006): 391-396.

29. Joiner A. "Tooth color: a review of the literature". Journal of Dentistry 32 (2004): 3-12.

30. Pitel M L. "Optimizing Your Shade-Matching Success: Tips, Tools, and Clinical Techniques". Dentistry Today 34.9 (2015): 116-118.

31. Jaju RA., et al. "Evaluating tooth color matching ability of dental students". Journal of Dental Education 74 (2010): 10021010.

32. Ristic I., et al. "Influence of Color Education and Training on Shade Matching Skills". Journal of Esthetic and Restorative Dentistry 28 (2016): 287-294.

33. Joshi R and Acharya J. "Shade Matching Ability of Dental Students Using Two Visual Light Sources". Nepal Medical College Journal 19 (2017): 24-26.

34. Chen H., et al. "A systematic review of visual and instrumental measurements for tooth shade matching". Quintessence International 43 (2012): 649-659.

35. Igiel C., et al. "Reliability of visual and instrumental color matching". Journal of Esthetic and Restorative Dentistry 29 (2017): 303-308.

36. Culic B., et al. "Clinical evaluation of a new software for shade matching". Optoelectronics and Advanced Materials-Rapid Communication 9 (2015): 1245-1250.

37. Oh WS., et al. "Digital computer matching of tooth color". Materials 3.6 (2010): 3694-3699.

38. Ahmad I. "Digital dental photography". Part 5: lighting". British Dental Journal 207.1 (2009): 13.

39. McLaren EA and Chang YY. "Photography and Photoshop®: simple tools and rules for effective and accurate communication". Inside Dentistry 2.8 (2006): 97-101.

40. Liu F. "Dental Digital Photography: From Dental Clinical Photography to Digital Smile Design”. Springer, (2019).

41. Anand D., et al. "Shade selection: Spectrophotometer vs. digital camera-A comparative in vitro study". Annals of Prosthodontics and Restorative Dentistry 2 (2016): 73-78. 
42. Schropp L. "Shade matching assisted by digital photography and computer software". Journal of Prosthodontics: Implant, Esthetic and Reconstructive Dentistry 18.3 (2009): 235-241.

43. Jarad F D., et al. "The use of digital imaging for color matching and communication in restorative dentistry". British Dental Journal 199.1 (2005): 43.

44. Hein S., et al. "eLABor_aid: a new approach to digital shade management. International Journal of Esthetic Dentistry 12.2 (2017): 186-202.

45. Zeighama S., et al. "Effect of Ceramic Thickness and Cement Color on Final Shade of All Ceramic Restorations: A Systematic Review". Scholars Academic Journal of Biosciences 5 (2017): 425-432.

46. Grah CL., et al. "Differences in color between fired porcelain and shade guides". International Journal of Prosthodontics 5 (1992): 510-514.

47. Pires LA., et al. "Effects of the type and thickness of ceramic, substrate, and cement on the optical color of a lithium disilicate ceramic". Journal of Prosthetic Dentistry 117 (2017): 144149.

48. Seghi RR., et al. "Spectrophotometric analysis of color differences between porcelain systems". Journal of Prosthetic Dentistry 56 (1986): 35-40.

49. Douglas RD and Przybylska M. "Predicting porcelain thickness required for dental shade matches". Journal of Prosthetic Dentistry 82 (1999): 143-149.

50. Stavridakis MM., et al. "Effect of different high-palladium metal-ceramic alloys on the color of opaque and dentin porcelain". Journal of Prosthetic Dentistry 92 (2004): 170-178.

51. Falahchai SM., et al. "Main Factors affecting the final color of ceramic restorations". Advances in Dentistry and Oral Health 5 (2017): 1-3.

52. Fasbinder DJ., et al. "A Clinical Evaluation of Chairside Lithium Disilicate CAD/CAM Crowns". Journal of the American Dental Association 141 (2014): 10-14.

53. Rauch A., et al. "Chair-side generated posterior monolithic lithium disilicate crowns: clinical survival after 6 years". Clinical Oral Investigations 21 (2017): 2083-2089.

54. Ballard E., et al. "Satisfaction of Dental Students, Faculty, and Patients with Tooth Shade-Matching Using a Spectrophotometer". Journal of Dental Education 81 (2017): 545-553.
55. Shah RJ., et al. "A Study of Patient Satisfaction with Maxillary Anterior Teeth Restorations and Desirable Esthetic Treatment Options". Journal of Medical and Dental Sciences 13 (2014): 7986.

\section{Assets from publication with us}

- Prompt Acknowledgement after receiving the article

- Thorough Double blinded peer review

- Rapid Publication

- Issue of Publication Certificate

- High visibility of your Published work

Website: www.actascientific.com/

Submit Article: www.actascientific.com/submission.php

Email us: editor@actascientific.com

Contact uS: +919182824667 